\title{
POSICIONAMENTO CIRÚRGICO EM CIRURGIA ROBÓTICA PEDIÁTRICA: RELATO DE EXPERIÊNCIA
}

\author{
Surgical Positioning in Pediatric Robotic Surgery: experience report \\ Posicionamiento quirúrgico en cirugía robótica pediátrica: relato de experiencia \\ Cecília da Silva Ângelo ${ }^{1}$ (D) Érica Adriana Lima da Silva ${ }^{2}$ (D), Anderson de Souza ${ }^{3 *}$ (D), Isabel Miranda Bonfim ${ }^{4}$, \\ Eduardo Henrique Giroud Joaquim ${ }^{5}$ (D), Maria Lúcia de Pinho Apezzato ${ }^{6}$ (1)
}

RESUMO: Objetivo: Relatar a experiência dos primeiros seis casos de cirurgia robótica pediátrica e a atuação dos enfermeiros especialistas em cirurgia robótica em uma instituição oncológica. Método: Trata-se de um relato de experiência vivenciada na prática clínica que fomentou a discussão acerca dos primeiros seis casos de cirurgia robótica pediátrica, ocorridos entre 2017 e 2019. Resultados: O processo de implantação da cirurgia robótica pediátrica começou em 2017, com dois casos iniciais (adrenalectomia e nefrectomia). Todos os casos foram acompanhados e executados na presença do cirurgião urologista proctor para auxiliar a cirurgiã oncológica pediátrica. O posicionamento cirúrgico depende do local a ser abordado e do procedimento robótico que será realizado. Conclusão: Identificou-se a necessidade de uma simulação realística com a participação de todos os membros da equipe para melhoria dos processos assistenciais e da educação permanente na formação da equipe.

Palavras-chave: Enfermagem perioperatória. Robótica. Pediatria.

ABSTRACT: Objective: To report the experience of the first six cases of pediatric robotic surgery and the role of nurses who are specialists in robotic surgery in an oncology institution. Method: This is an account of experience report in clinical practice which encouraged the discussion about the first six cases of pediatric robotic surgery, which occurred between 2017 and 2019. Results: The process of implantation of pediatric robotic surgery started in 2017, with two initial cases (adrenalectomy and nephrectomy). All cases were followed up and performed in the presence of the proctor urologist surgeon to assist the pediatric oncology surgeon. Surgical positioning depends on the location to be approached and the robotic procedure that will be performed. Conclusion: The need for a realistic simulations with the participation of all team members to improve the care processes and permanent education in team formation was identified.

Keywords: Perioperative nursing. Robotics. Pediatrics.

RESUMEN: Objetivo: informar la experiencia de los primeros seis casos de cirugía robótica pediátrica y el papel de las enfermeras especialistas en cirugía robótica en una institución de oncología. Método: Este es un relato de la experiencia en la práctica clínica, que fomentó la discusión sobre los primeros seis casos de cirugía robótica pediátrica, que ocurrieron entre 2017 y 2019. Resultados: El proceso de implantación de cirugía robótica pediátrica comenzó en 2017, con dos casos iniciales (adrenalectomía y nefrectomía). Todos los casos fueron seguidos y realizados en presencia del cirujano urólogo supervisor para ayudar al cirujano oncólogo pediátrico. El posicionamiento quirúrgico depende de la ubicación a abordar y del procedimiento robótico que se realizará. Conclusión: Se identificó la necesidad de una simulación realista con la participación de todos los miembros del equipo para mejorar los procesos de atención y la educación permanente en la formación del equipo.

Palabras clave: Enfermería perioperatoria. Robótica. Pediatría.

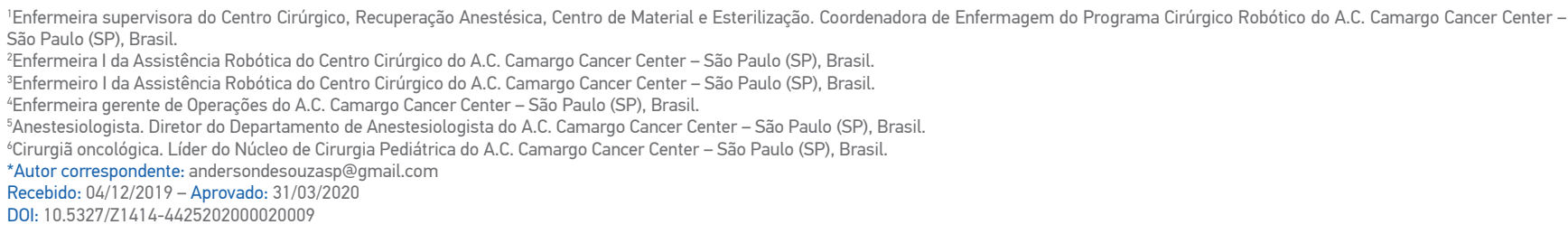




\section{INTRODUÇÃo}

O tratamento da neoplasia infantojuvenil tem avançado nas últimas décadas em virtude das técnicas de diagnóstico precoce e da evolução dos métodos terapêuticos. Cada planejamento terapêutico determina as chances de cura do paciente e tem particularidades, recomendações, limites e benefícios, além de possíveis complicações ${ }^{1}$.

A tecnologia cirúrgica avança rapidamente, tornando-se uma prática comum. O sistema cirúrgico robótico Da

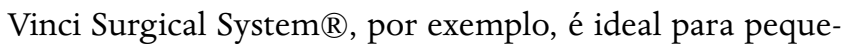
nos espaços, de modo que a cirurgia assistida por robô está se estendendo até a especialidade pediátrica. Os benefícios documentados da cirurgia robótica em pediatria, desde o primeiro caso no ano 2000, são basicamente os mesmos que a cirurgia minimamente invasiva por meio da laparoscopia, com menor tempo de internação, menor sangramento, menor incidência de dor, recuperação mais rápida e melhor resultado estético ${ }^{2,3}$.

O posicionamento cirúrgico em cirurgia robótica pediátrica assemelha-se ao dos pacientes adultos, no entanto a equipe multidisciplinar deve levar em consideração o tamanho do paciente e entender o contexto da plataforma robótica. Cada procedimento robótico requer diferentes posicionamentos e a utilização de dispositivos adequados para auxiliar no posicionamento do paciente. É importante para o enfermeiro perioperatório compreender as instruções mais seguras do posicionamento do paciente em cirurgia robótica ${ }^{4}$.

A segurança do paciente e a eficiência do procedimento podem ser comprometidas se o enfermeiro perioperatório não for capacitado quanto aos cuidados para pacientes submetidos aos procedimentos robóticos ou não tiver conhecimento técnico/ científico da tecnologia. Estudos demonstraram certa preocupação na capacitação e na formação da equipe de enfermagem em cirurgia robótica. Dessa forma, as instituições ofereceram um programa de treinamento para os enfermeiros perioperatórios envolvidos na cirurgia robótica, proporcionando competência à prática desses profissionais, redução de complicações, promoção de resultados positivos para a assistência de enfermagem e melhora de seus indicadores de qualidade ${ }^{5-7}$.

O presente trabalho visa relatar a experiência dos primeiros seis casos de cirurgia robótica pediátrica e a atuação dos enfermeiros especialistas em cirurgia robótica em uma instituição oncológica.
A cirurgia minimamente invasiva tem sido um grande desafio para o profissional da enfermagem que atua com equipamentos de alta tecnologia e inovações em técnicas cirúrgicas com aprimoramentos exponenciais. Dessa forma, o engajamento e o empoderamento desse profissional com a equipe multidisciplinar são essenciais para uma assistência assertiva, com qualidade e garantia da credibilidade em seus conhecimentos teóricos e práticos. A tecnologia cirúrgica minimamente invasiva pela modalidade robótica, cada vez mais, faz com que o profissional de enfermagem se atualize e se aproxime dos especialistas dessa área, fortalecendo esse tipo de estudo e impulsionando os profissionais na busca de aprimoramento contínuo.

\section{OBJETIVO}

Relatar a experiência dos primeiros seis casos de cirurgia robótica pediátrica e a atuação dos enfermeiros especialistas em cirurgia robótica em uma instituição oncológica.

\section{MÉTODO}

Trata-se de um relato de experiência vivenciada na prática clínica que fomentou a discussão acerca dos primeiros seis casos de cirurgia robótica pediátrica e da atuação dos enfermeiros especialistas em cirurgia robótica em uma instituição oncológica, entre os anos de 2017 e 2019.

O cenário utilizado está inserido em uma instituição oncológica de grande porte, filantrópica, localizada no município de São Paulo (SP). O centro cirúrgico (CC) possui 14 salas cirúrgicas e sua média é de 1.000 cirurgias/mês. Desses 1.000 procedimentos, cerca de 40 são realizados com a tecnologia robótica.

O processo de implantação da cirurgia robótica pediátrica começou em 2017, com dois casos iniciais, sendo uma adrenalectomia e uma nefrectomia. Os outros quatro casos subsequentes realizados foram: nefrectomia, cistoprostatectomia, histerectomia e cistectomia. Todos foram acompanhados e executados na presença do cirurgião urologista proctor para auxiliar a cirurgiã oncológica pediátrica.

O processo de implantação da cirurgia robótica pediátrica é desafiador. Inicialmente, adotaram-se os protocolos específicos, bem delineados e de caráter multidisciplinar, que norteiam os profissionais a realizarem sua função de maneira segura. 


\section{RESULTADOS}

Na véspera da cirurgia, os enfermeiros do programa robótico reuniram os materiais e equipamentos para atender a essa modalidade cirúrgica. Além disso, houve uma simulação realística com a participação da equipe cirúrgica, composta de: enfermeiro, técnico de enfermagem (circulante de sala), instrumentador, cirurgião e anestesiologista. Cada membro da equipe apresentou excelente desempenho nas atribuições executadas. Soma-se a importância da instituição em ter os dispositivos adequados para auxiliar no posicionamento cirúrgico em cirurgia robótica pediátrica.

Nos casos iniciais, posicionaram-se os pacientes submetidos à adrenalectomia e à nefrectomia em decúbito lateral, sob anestesia geral inalatória e venosa. O enfermeiro perioperatório aplicou cobertura de adesivo de redistribuição de pressão nas proeminências ósseas que ficaram apoiadas na mesa cirúrgica. Sustentou-se a cabeça no polímero viscoelástico cefálico em forma de halo, que é utilizado para repousar a cabeça e evitar pressão no pavilhão auricular externo. Um membro superior ficou apoiado no polímero viscoelástico sobre a mesa cirúrgica e o outro paralelo sobre uma espuma piramidal.

A instabilidade do paciente é corrigida flexionando o membro inferior apoiado na mesa cirúrgica e mantendo o superior esticado em uma espuma piramidal, reduzindo a pressão ocasionada pelo peso do membro superior no inferior. A completa estabilização do paciente em decúbito lateral é obtida aplicando uma cobertura de adesivo de redistribuição na crista ilíaca e uma tira de esparadrapo com fixação na mesa cirúrgica.

Nas cirurgias de cistoprostatectomia, histerectomia e cistectomia utilizou-se a posição de Trendelenburg, na qual o paciente é colocado em decúbito dorsal na mesa cirúrgica e recebe uma inclinação, onde o tronco e a cabeça ficam em plano inferior. Com essa inclinação, a tendência natural é que o paciente deslize em direção à cabeceira da mesa. Portanto, houve a necessidade de realizar a fixação em cima do tórax com uma espuma piramidal e com esparadrapo para evitar qualquer movimentação acidental no intraoperatório. Os membros superiores ficaram apoiados na mesa cirúrgica, a região do quadril ficou apoiada na borda da articulação da mesa e os membros inferiores flexionados, facilitando o posicionamento. O enfermeiro perioperatório deve realizar uma revisão do posicionamento após o docking do robô (aproximação à mesa cirúrgica).

Certamente o posicionamento cirúrgico depende do local a ser abordado e do procedimento robótico que será realizado. É fundamental que o enfermeiro especialista em cirurgia robótica esteja atento aos detalhes do procedimento e aos dispositivos adequados para o posicionamento cirúrgico. Em cada paciente, utilizaram-se as técnicas de posicionamento, respeitando a afecção e suas condições anatômicas. A ampla experiência do profissional enfermeiro em cirurgias robóticas teve resultados iniciais positivos nessa nova modalidade cirúrgica.

\section{DISCUSSÃO}

Com base na literatura disponível, o papel da enfermagem em cirurgia robótica tem um impacto positivo tanto no paciente quanto na equipe multidisciplinar, por meio de suas competências, aliadas à melhoria dos processos assistenciais e da educação permanente na formação da equipe $e^{6,8,9}$.

Estudos reforçam que um dos fatores significativos e desafiadores é o posicionamento do paciente em cirurgia robótica, que é de responsabilidade compartilhada entre o enfermeiro perioperatório, o anestesiologista e o cirurgião ${ }^{10}$. Em outro estudo, os autores destacam a importância de cada membro da equipe em reconhecer seu papel e sua responsabilidade na cirurgia robótica ${ }^{8}$.

Um estudo epidemiológico realizado na cidade de São Paulo reportou uma incidência acumulativa de lesão por pressão (LP) de 21,4\% (IC95\% 1,8-42,8) em crianças hospitalizadas tratadas no CC, com amostra de 229 pacientes, com idades entre 30 dias e 18 anos. Nesse estudo, além dos fatores conhecidos, como imobilidade, umidade, tempo cirúrgico, variação de temperatura e índice de massa corpórea, os fatores de risco associados foram a sedação/analgesia $(\mathrm{p}=0,04)$ e os tratamentos de cuidado intensivo, por exemplo, a ventilação mecânica $(\mathrm{p}=0,001)^{11}$.

No caso dos pacientes infantojuvenis, observações empíricas têm indicado que de 20 a 40 minutos de isquemia em proeminências ósseas são suficientes para o desenvolvimento de injúria tecidual, com a consequente formação de $\mathrm{LP}^{11}$.

Para manter a segurança desses pacientes, é importante ter dispositivos adequados para essa modalidade cirúrgica: além de inspecionar a pele, as áreas de proeminências ósseas, respeitar o alinhamento corporal anatômico e documentar qualquer alteração na integridade da pele na ficha de sistematização da assistência de enfermagem perioperatória (SAEP) ${ }^{4,7}$.

Outro estudo, realizado nos Estados Unidos, avaliou os resultados clínicos e os custos associados à cirurgia robótica em população pediátrica de 43 instituições participantes da pesquisa, das quais $51 \%$ tiveram casos em pediatria. 
Os achados indicaram estadia hospitalar mais curta, porém com maior custo $^{12}$.

A cirurgia robótica é uma tecnologia que demonstrou ser segura e eficaz para os procedimentos pediátricos, principalmente para ressecções de tumores ${ }^{13}$.

Estudos prospectivos mostraram que a evolução do procedimento robótico oferecerá manejos alternativos no tratamento de pacientes pediátricos, com melhorias no atendimento e na qualidade de vida ${ }^{14}$.

\section{CONSIDERAÇÕES FINAIS}

Este relato de experiência possibilitou reconhecer as atribuições do enfermeiro e dos membros da equipe cirúrgica em cirurgia robótica pediátrica. Entretanto, identificou-se a necessidade de uma simulação realística com a participação de todos os membros da equipe para melhoria dos processos assistenciais e da educação permanente na formação da equipe.

Outra particularidade observada no estudo é a necessidade de que a instituição tenha dispositivos adequados para auxiliar no posicionamento do paciente em cirurgia robótica pediátrica. Portanto a utilização de dispositivos adequados deve ser entendida como um parâmetro de boas práticas da enfermagem e da equipe multidisciplinar que pode contribuir, na prevenção de LP decorrente do posicionamento cirúrgico.

A literatura revelou-se escassa. Desse modo, sugere-se que sejam desenvolvidos novos estudos para o assunto em questão, visando à atuação em uma prática clínica com excelência e ao delineamento do papel do enfermeiro em cirurgia robótica.

\section{REFERÊNCIAS}

1. Benedetti GMS, Garanhani ML, Sales CA. 0 tratamento do câncer infantojuvenil: desvelando as vivências dos pais. Rev Latino-am Enferm. 2014;22(3):425-31. https://doi.org/10.1590/0104-1169.3264.2433

2. Bruns NE, Soldes OS, Ponsky TA. Robotic surgery may not "make the cut" in pediatrics. Front Pediatr. 2015;(3): 10-3. http://doi.org/10.3389/ fped.2015.00010

3. Harrington S, Simmons K, Thomas C, Scully S. Pediatric laparoscopy. AORN J. 2008;88(2):211-40. https://dx.doi.org/10.1016/j. aorn.2008.04.002

4. Hortman C, Chung S. Positioning considerations in robotic surgery. AORN J. 2015;102(4):434-40. http://doi.org/10.1016/j.aorn.2015.07.008

5. Sarmanian JD. Robot-Assisted Thoracic Surgery (RATS): perioperative nursing professional development program. AORN J. 2015;102(3):24153. http://dx.doi.org/10.1016/j.aorn.2015.06.013

6. Sousa CS, Bispo DM, Cunha AL. Capacitação em cirurgia robótica no programa de residência em enfermagem perioperatória. Rev SOBECC. 2016;21(4):198-202. https://doi.org/10.5327/ Z1414-4425201600040004

7. Angelo CS, Pachioni CFM, Joaquim EHG, Silva EAL, Santos GG, Bonfim IM, et al. Efetividade do protocolo prevenção de lesões de pele em cirurgias urológicas robóticas. Rev SOBECC. 2017;22(3):15260. https://doi.org/10.5327/Z1414-4425201700030006
8. Martins RC, Trevilato DD, Jost MT, Caregnato RCA. Atuação da enfermagemem cirurgias robóticas: revisão integrativa. RevBras Enferm. 2019;72(3):832-8. https://doi.org/10.1590/0034-7167-2018-0426

9. Pinto EV, Lunardi LS, Treviso P, Botene DZA. Atuação do enfermeiro na cirurgia robótica: desafios e perspectivas. Rev SOBECC. 2018;23(1):4351. https://doi.org/10.5327/Z1414-4425201800010008

10. Myklebust MV, Storheim H, Hartvik M, DysvikE. Anesthesia professional's perspectives of teamwork during robotic-assisted surgery. AORN J. 2020;111(1):87-96. http://dx.doi.org/10.1002/aorn.12897

11. Pellegrino DMS, Blanes L, Chacon JMF, Ferreira LM. Prevalence and incidence of pressure injuries in pediatric hospitals in the city of São Paulo, 51, Brazil. Journ Tissue Viability. 2017;26(4):241-5. https:// doi.org/10.1016/j.jtv.2017.07.001

12. Mahida JB, Cooper JN, Hernz D, Diefenbach KA, Deans KJ, Minneci PC, et al. Utilization and costs associated with robotic surgery in children. $J$ Surg Res. 2015;199(1):169-76.https://dx.doi.org/10.1016/j.jss.2015.04.087

13. Meehan JJ. Robotic surgery for pediatric tumors. Cancer J. 2013;19(2):183-8. https://doi.org/10.1097/PPO.0b013e318289486c

14. Lambert G, Fourcade L, Centi J, Fredon F, Braik K, Szwarc C, et al. How to successfully implement a robotic pediatric surgery program: lessons learned after 96 procedures. Surg Endosc. 2013;27(6):213744. https://doi.org/10.1007/s00464-012-2729-y 